\title{
Extraction of accurate cytoskeletal actin velocity distributions from noisy measurements
}

\author{
Cayla M. Miller, Elgin Korkmazhan, ${ }^{*}$ and Alexander R. Dunn ${ }^{\dagger}$ \\ Department of Chemical Engineering, Stanford University, Stanford, CA, USA
}

(Dated: August 12, 2020)

\begin{abstract}
Dynamic remodeling of the actin cytoskeleton allows cells to migrate, change shape, and exert mechanical forces on their surroundings. How the complex dynamical behavior of the cytoskeleton arises from the interactions of its molecular components remains incompletely understood. Tracking the movement of individual actin filaments in living cells can in principle provide a powerful means of addressing this question. However, single-molecule fluorescence imaging measurements that could provide this information are limited by low signal-to-noise ratios, with the result that the localization errors for individual fluorophore fiducials attached to filamentous (F)-actin are comparable to the distances traveled by actin filaments between measurements. In this study we tracked the movement F-actin labeled with single-molecule densities of the fluorogenic label SiR-actin in primary fibroblasts and endothelial cells. We then used a Bayesian statistical approach to estimate true, underlying actin filament velocity distributions from the tracks of individual actin-associated fluorophores along with quantified localization uncertainties. This analysis approach is broadly applicable to inferring statistical pairwise distance distributions arising from noisy point localization measurements such as occur in superresolution microscopy. We found that F-actin velocity distributions were better described by a statistical jump process, in which filaments exist in mechanical equilibria punctuated by abrupt, jump-like movements, than by models incorporating combinations of diffusive motion and drift. A model with exponentially distributed time- and length-scales for filament jumps recapitulated F-actin velocity distributions measured for the cell cortex, integrin-based adhesions, and actin stress fibers, indicating that a common physical model can potentially describe F-actin dynamics in a variety of cellular contexts.
\end{abstract}

\section{INTRODUCTION}

The actin cytoskeleton is both dynamic and mechanically robust, allowing it to simultaneously define cell shape while facilitating membrane protrusion and cell migration. These roles require cytoskeletal assemblies with distinct physical properties[1-4]. Two such examples are the actin cortex, a thin contractile mesh immediately beneath the cell membrane that helps to maintain cell shape[5], and stress fibers, thick bundles of crosslinked actin and myosin that transmit force to the extracellular matrix (ECM) through focal adhesions[6]. Additional actin-based structures (lamellipodia, filopodia, podosomes, invadopodia) are formed in specific contexts to enable cell migration, sensing, and ECM remodeling[7]. These F-actin based structures generate force through a combination of pushing forces resulting from F-actin polymerization and pulling forces generated by motor proteins, most prominently non-muscle myosin II. Imbalances in these push-pull forces drive localized F-actin flows, which thus report on the local dynamics of cytoskeletal remodeling and cellular force generation[8].

Experiments quantifying local F-actin flow velocities in the leading edge of migrating cells have yielded key insights into how cell shape, migration, and force transmission arise at the molecular level. Early work

\footnotetext{
* Biophysics Program, Stanford University, Stanford, CA, USA

$\dagger$ alex.dunn@stanford.edu
}

examining $\mathrm{F}$-actin dynamics noted rapid actin turnover and rearward flow from the cell edge toward the cell body in the lamellipodia of both keratocytes and fibroblasts $[9$, 10], a phenomenon termed retrograde flow that has since been observed and characterized in many cell types[2, 3, 11-14]. Speckle microscopy established the lamellipodia and lamella as distinct regions with unique F-actin retrograde flow velocities and polymerization kinetics, pointing to specialized roles in ECM exploration and persistent advancement of the cell's leading edge, respectively $[2,15]$. Kymograph-based analysis of $\mathrm{F}$-actin velocities in fish keratocytes demonstrated that myosin II inhibition can speed or slow retrograde flow, depending on the force balance in an individual cell[16]. Multiple studies have also shown that actin flows are slowed locally over integrin-based cellular adhesion complexes, termed focal adhesions, a phenomenon that reflects localized traction generation[17-20]. These and other studies support a consensus understanding, termed the molecular clutch model, in which rearward flow of F-actin is coupled by frictional slippage to focal adhesions in order to exert traction on the cell's surroundings[17, 2123]. Fewer studies have focused on actin dynamics under the cell body, where directed, vectoral F-actin flow is less apparent[24, 25]. This knowledge gap is potentially important, as the lamellipodia and lamella constitute a small fraction of the cell's surface in most cell types.

A separate body of work has used biophysical measurements to infer the mechanical properties of the cytoskeleton. Experiments that used magnetic beads attached to the cell's exterior to exert indirect forces on the cell cytoskeleton support a model in which cells, and 
by extension the cytoskeleton, can be described as soft, glassy materials close to the glass transition point, or else as gels[26-28]. In these models, actin filaments form a metastable crosslinked network that rearranges over a broad range of timescales. This general view is supported by a variety of measurements, including optical trap experiments[29], atomic force microscopy measurements, and a variety of other techniques (reviewed in[30]).

It is striking that the large majority of studies characterizing the cytoskeleton have quantified bulk, or averaged, F-actin dynamics as opposed to those of individual filaments. Cell rheological measurements, as discussed above, necessarily average over large numbers of filaments. The large majority of F-actin tracking measurements, such as those supporting the molecular clutch model, use fluorescent fiducial densities that are too high to distinguish the motion of individual filaments[31]. Notably, a few studies used sufficiently low concentrations of $\mathrm{F}$-actin fiducials to track single filaments[20, 32, 33]. However, where these data have been analyzed to give short-timescale dynamics of individual filaments, the focus has remained on a relatively small number of filaments near the cell edge[20].

Recent results from our laboratory implied that Factin attached to integrin-based adhesions does not undergo continuous retrograde flow, but instead moves with a discontinuous stick-slip motion[34]. This picture contrasts with the canonical view of continuous, retrograde F-actin flow arising from the molecular clutch model. Although it is not a feature of most molecular clutch models, stick-slip behavior would be consistent with a description of the cytoskeleton as a gel or glass[28, 35]. However, as discussed above, to our knowledge a suitable measurement of single-filament dynamics in living cells has not been reported.

Here, we present a novel analysis that uses prior information about the localization error and Bayesian inference to estimate true underlying velocity distributions from noisy fiducial tracking data. Our measurements revealed details of distinct velocity distributions for $\mathrm{F}$-actin populations inside and outside stress fibers, and also showed how the velocity distributions evolve over sub-minute timescales. At short timescales $(<10 \mathrm{~s})$, our analysis revealed the presence of slowly moving $\mathrm{F}$-actin, which was previously difficult to discern due to measurement noise, along with a long tail of faster-moving filaments. At longer timescales (10s of seconds), the velocity distribution became more narrowly distributed around a peak velocity. We propose one physical model that is consistent with these observations, namely a statistical jump process that describes the movement of individual actin filaments.
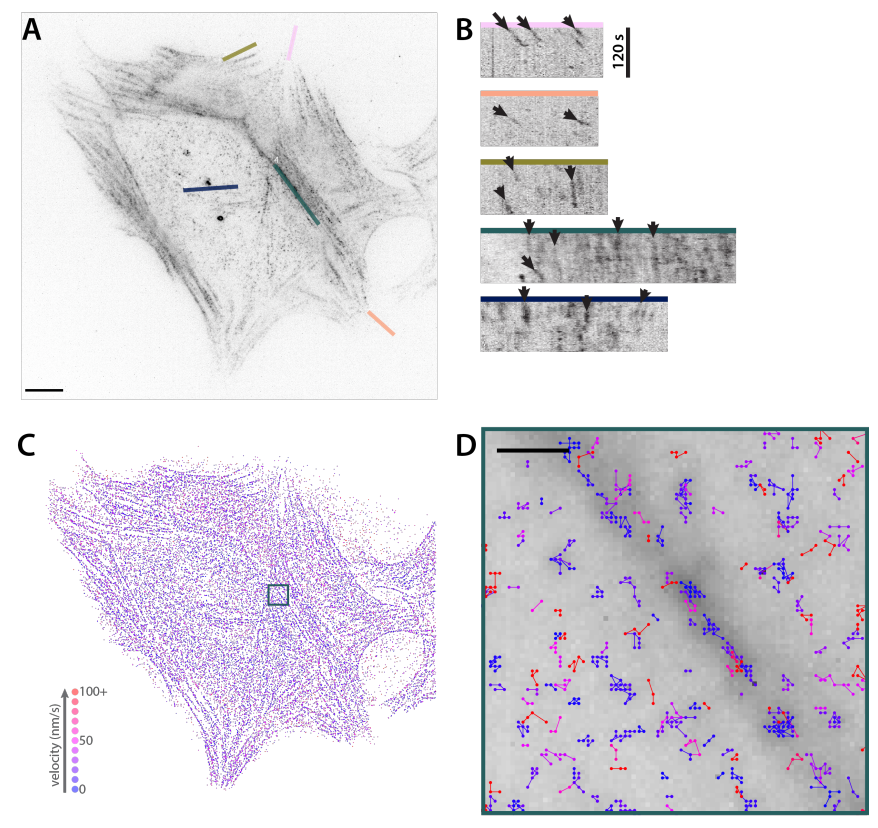

FIG. 1. SiR-Actin labelling reveals variation in $\mathbf{F}$-actin velocities in fibroblasts. (A) An HFF labelled with a low dose of SIR-actin shows sparse labelling of both stress fibers and the cortex. Scale bar is $10 \mu \mathrm{m}$. (B) Kymographs showing actin motion in the cell in A over $120 \mathrm{~s}$, showing fast motion at the cell edge (pink and peach), slower motion closer to the cell body (olive), and nearly-stationary particles in stress fibers (teal and blue). (C) Single molecule tracking via QFSM for the same cell over the same $120 \mathrm{~s}$ interval. The first point of each track is shown, color-coded by its apparent velocity. (D) Close up of the boxed region in C, showing all points of the tracks in and surrounding a stress fiber. The tracks are overlaid over the mean intensity projection of the time series. Scale bar is $1 \mu \mathrm{m}$.

\section{RESULTS}

We quantified the motion of individual F-actin filaments in human foreskin fibroblasts (HFFs) by sparsely labelling with very low concentrations of SiRactin, a probe whose fluorescence increases 100-fold when bound to F-actin. The use of SiR-actin provides several advantages: compatibility with many cell types without genetic engineering, live cell permeability without harsh electroporation or microinjection, and binding-specific fluorescence resulting in low fluorescence background[36]. The cells were imaged using TIRF microscopy, limiting the fluorescence excitation field to the basal surface $(<$ $200 \mathrm{~nm}$ ) of the cell. The F-actin fiducials marked by SiR-actin were imaged every two seconds for a total of two minutes (Video S1). Time-lapse recordings revealed a small population of fast-moving, highly directed actin flowing retrograde at the cell edge (Figure 1, A-B, pink, peach, and olive kymographs). While important in the context of lamellipodial extension, this population constitutes a small minority of the F-actin in most adherent cell types, and moreover has been intensively 
studied[2, 3, 11-16, 18-20]. We therefore focused on Factin populations under the cell body.

F-actin in stress fibers moved slowly relative to lamellipodial F-actin (Figure 1, A-B, teal and blue kymographs), as previously shown[23, 25]. We observed coherent motion of fiducials in the same stress fiber, and in many cases approximately constant velocities over the observation window, suggestive of locally coordinated flows. Finally, we observed a large pool of F-actin belonging to neither the slow-moving stress fiber population nor the fast-moving cell-edge population. This population represents the cortical actin network. It has been shown that this network is important for transmitting tension along the contour of the cell membrane[37], and exhibits a wide range of speeds in yeast $(0-1.9 \mathrm{um} / \mathrm{s})[38]$. However, the motion of this population is not well-characterized in mammalian cells.

\section{A. Accurate F-actin velocity distribution can be inferred from noisy measurements}

We used quantitative fluorescent speckle microscopy (QFSM)[39] to track SiR-actin puncta in all areas of the cell (Figure 1, C-D). QFSM showed that stress fibers contain slow moving, longer lasting tracks, consistent with the anecdotal evidence from kymographs. However, velocities calculated from raw QFSM tracks are compromised by a potentially serious overestimation of the displacement between time steps (Figure 2). For a fluorophore with a given localization error $\sigma_{x y}$ in the imaging plane which moves a true distance $s$ between frames, the measured distance $d$ is sampled from a noncentral $\chi$ distribution with 2 degrees of freedom and a mean given by $\sqrt{s^{2}+4 \sigma_{x y}^{2}}(\mathrm{~A})$.

Consequently, for small displacements, the localization error overwhelms the measured distance. Figure 2, AB shows simulations of measured distances between two positions with a true distance $s=5 \sigma_{x y}$, as well as for a true distance of $s=0$ (i.e. a stationary point) with $\sigma_{x y}=1$. In both cases, the distribution of measured distances was in good agreement the analytical solution given by a noncentral $\chi$ distribution. For a stationary particle $(s=0)$, all measurements are greater than the true displacement of zero, giving a peaked distance distribution (with peak at $\sqrt{2} \sigma_{x y}$ ). At the longer distance, the average measured distance is roughly correct, but any individual measurement is drawn from a broad distribution. At both true distances, the distribution of measured distances is broader than the (Dirac delta-function) true distribution.

In order to quantitatively characterize the localization error in our speckle microscopy videos, we treated chemically fixed cells with SiR-actin and tracked the actin fiducials through time in the same way as our live cell samples. The resulting distribution of measured displacements was peaked at non-zero values, as predicted (Figure 2C). We fit this distribution of measured distances to a noncentral $\chi$ with $s=0$. Here and hereafter we report maximum likelihood estimates for fit parameters; MLE allows us to maximally leverage the information in tracks ( $\mathrm{n}=39,582$ tracks in fixed cells), rather than fitting to the binned histogram. This singleparameter fit gave us $\sigma_{x y}=47 \mathrm{~nm}$ for the localization error in our system (Figure 2C). Over varying timescales of analysis $(2-40 \mathrm{~s})$ using constant, $0.3 \mathrm{~s}$ exposures, the distribution of measured distances in fixed cells was similar, but with a small enrichment of longer step sizes over longer timescales (Figure S1). This effect may stem from a number of factors including incomplete fixation, in-plane or out-of-plane sample drift, or algorithm mismatches of fiducials. However, the effect is relatively modest, and we use the best estimate of our localization error ( $47 \mathrm{~nm}$ at 2 to $10 \mathrm{~s}$ time delays) in our downstream workflow (Figure $2 \mathrm{G}$ ).

With this information, we can consider more complex cases, where the true distance, $s$, is not constant, but is a random variable sampled from some distribution, $f_{s}(s)$. In this case, the distribution of measured displacements depends on two factors: the probability distribution of the true distances, $f_{s}(s)$, and the conditional probability, $f_{d}(d \mid s)$ of measuring a displacement, $d$, given a true underlying distance, $s$. We use Bayes' theorem to infer the true displacement distribution from the distribution of measured displacements:

$$
f_{d}(d)=\int_{0}^{\infty} f_{d}(d \mid s=S) f_{s}(S) d S
$$

where $f_{d}(d \mid s=S) \sim \chi_{N C}\left(S, \sigma_{x y}\right)$, where $\chi_{N C}$ is the noncentral $\chi$ distribution. Therefore, for a measured distribution of distances and knowledge of the localization error $\left(\sigma_{x y}\right)$, one can infer the distribution of true distances. It is important to note that the fitting is not model-agnostic, and requires an assumed functional form for $f_{s}(s)$. However, the fitting can be quickly solved numerically, and fitting to various distributions is trivial.

As an illustrative example, we fit simulated F-actin track data with true displacements drawn from a Weibull distribution, with Gaussian localization error (Figure 2, D-F). The Weibull distribution yields either a longtailed exponential or peaked shape, depending on the choice of parameters, and thus is capable of capturing a wide range of physical behaviors at a phenomenological level. For a case where average distance, variance, and localization error are all the same magnitude, (Weibull shape $=1.5$, scale $=\sigma_{x y}$ ), the true and measured distances are shown together in Figure 2E. We fit the simulated distribution in $\mathrm{E}$ to equation 1 , with $f_{s} \sim \operatorname{Wbl}(k, \theta)$, and the localization error fixed. The fit matched the simulated data well (E), and the best fit parameters accurately captured the true distribution $(\mathrm{F})$. We used a similar workflow to infer true distributions from our live cell $\mathrm{F}$-actin displacements and the measured value of $\sigma_{x y}$ from our fixed cell measurement (Figure 2G). 
A
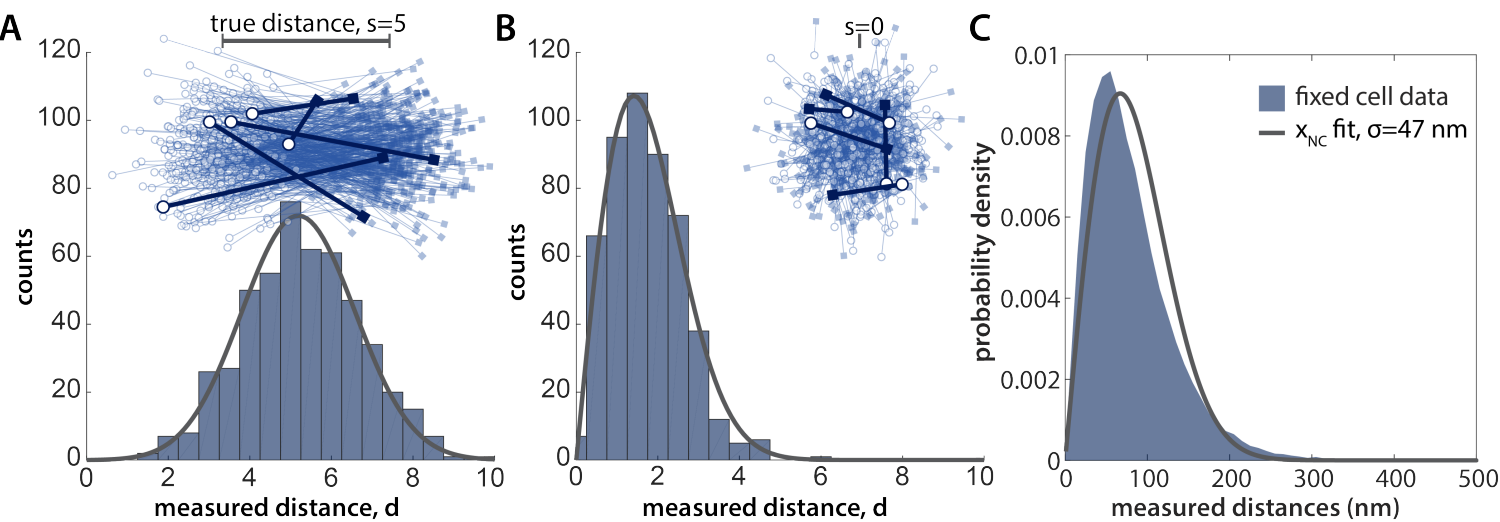

D

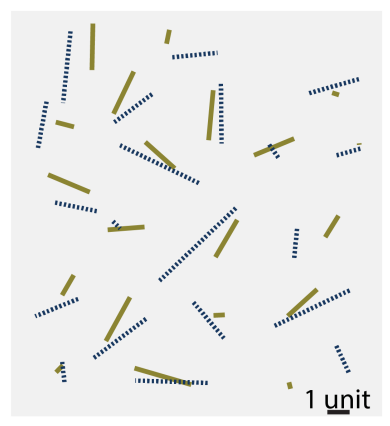

E
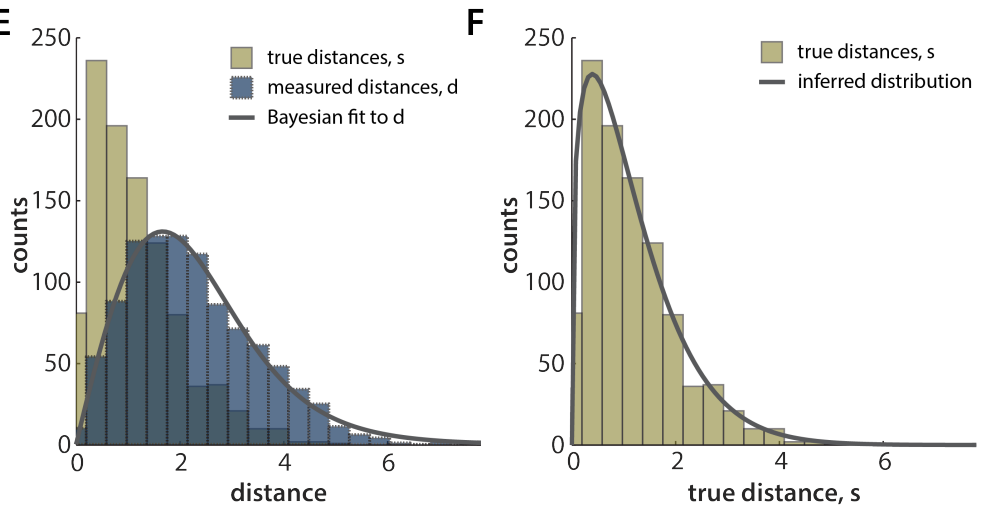

G

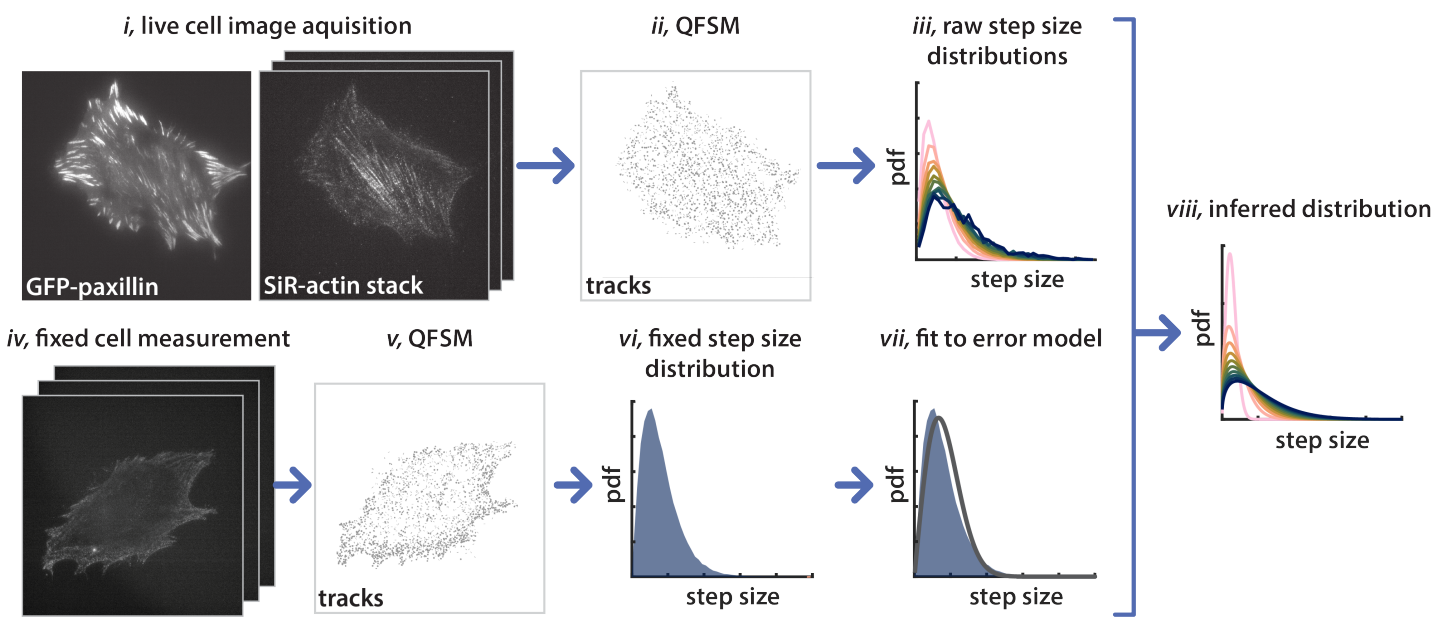

FIG. 2. Bayesian fitting to reconstruct a distribution of true distances that are otherwise obfuscated by localization error. (A) Simulated pairs of points sampled around two true locations with a distance $(s=5)$ greater than the localization error $\left(\sigma_{x y}=1\right)$. The first point of each distance measurement is marked by a hollow circle, and the second point by a solid square. Five representative pairs are shown in heavier weight. The histograms of measured distances (bars) and the predicted distribution of measured distances given by a noncentral $\chi$ (solid line) are shown below. (B) Simulated pairs of points with a true distance of 0 , and the corresponding histogram of measured distances and the analytically predicted distribution. (C) The distribution of displacements measured in fixed cells (over $10 \mathrm{~s}$ ) fit to a noncentral $\chi$ distribution with $\mathrm{s}=0$. (D) Simulated frame-to-frame motion of 20 simulated tracks, showing both the true movements of each punctum between the two frames drawn from a Weibull distribution (shape $=1.5$, scale $=\sigma_{x y}$; solid lines) and the error-laden measured movement (dashed lines). (E) The distribution of true distances (olive) and measured distances (blue) for 1000 such steps. The best fit to the measured distribution, determined by maximum likelihood estimation, is shown in gray. (F) The resulting inferred distribution from the fit in E (gray), overlaid with the true distribution of distances (olive bars). G) This method is applied to live-cell measurements: i,iv. Images of both GFP-paxillin-marked adhesions and SiR-actin are acquired on a TIRF microscope. ii,v. QFSM[39] is used to identify puncta and tracks through time. iii,vi. From the tracks, the displacements of individual puncta gives a distribution of velocities across the whole cell. This process is repeated for live (i-iii) and fixed (iv-vi) cells. vii, viii. Using the fixed cell data as a measure of the localization error, we can infer the true step size distribution from live-cell measurements. 


\section{B. Fit velocity distributions show distinct physical properties for stress fibers and cortical actin}

Many previous F-actin tracking measurements have avoided the high errors associated with slow actin speeds by focusing on regions of the cell where movements are fast, by filtering out nearly-stationary particles in a population, or by imaging over longer timescales (minutes to tens of minutes), where the displacements become larger. In our study, we used non-subsequent frames to measure displacements over a number of timescales ranging from 2 to $40 \mathrm{~s}$ with a constant exposure of $0.3 \mathrm{~s}$ per frame. During our analysis, we sorted F-actin fiducials into four mutually-exclusive populations based on their location within or outside stress fibers, and over or not over focal adhesions. For all of these subpopulations the peak of the distribution moved rightward and the distribution broadened at longer timescales (Figure 3A). However, because of the localization error, the shapes and magnitudes of these distributions cannot be directly interpreted.

We inferred true velocity distributions by fitting our live cell measurements using equation 1 , with $\sigma_{x y}=47$ $\mathrm{nm}$. There are many functional forms to choose from for $f_{s}(s)$, but for parsimony, we began with the simplest: the exponential, Gaussian, and Rayleigh distributions. These reflect actin movements driven by a single-step Poisson process, averaged movements described by the central limit theorem, and purely diffusive 2D motion, respectively. An exponential distribution fit F-actin displacements in stress fibers reasonably well over all timescales examined here $(2-40 \mathrm{~s})$, but did not fit the cortical actin population at longer timescales, Figure S2). Because our distance data are constrained to be positive, we fit to a folded Gaussian, rather than true Gaussian distribution. This distribution fit most of the data reasonably well but failed to capture the tails of the distribution (Figure S3). If the true distribution were governed by a purely diffusive process, we would expect the distribution of step sizes to follow a Rayleigh distribution. This distribution also captured a portion of the populations, but did not fit the F-actin tracks in stress fibers (Figure S4).

We next tested the Weibull distribution, a twoparameter model that interpolates between the exponential (Poisson process) and Rayleigh (diffusive) distributions:

$$
f(s)=\frac{k}{\lambda}\left(\frac{x}{\lambda}\right)^{k-1} e^{(x / \lambda)^{k}}
$$

where $k$ is the shape parameter, and $\lambda$ is the scale parameter. This distribution fit the data well for all four populations over all timescales, and the exponential character proved sufficient to capture the tails of the data. Comparing the likelihood ratios of these fits with the next best fitting, the folded Gaussian, revealed that the best-fit Weibull is $10^{117}-10^{3238}$ more likely than the best-fit folded Gaussian for each population (Table S1).
We scaled the fit distance distributions by the observation time to generate velocity distributions for all timescales (Figure 3B). This affects only the scale parameter, not the shape parameter, of the distribution. The shape parameter, $k$, from these fits showed a relatively constant value around 1.4 , indicating that the behavior on these timescales lies between exponentiallike behavior $(\mathrm{k}=1)$ and Rayleigh-like behavior $(\mathrm{k}=2)$ (Figure 3C). The scale parameter, however, was inversely related to timescale. Physically, F-actin motion was thus neither purely diffusive nor Poissonian, and showed average velocities that varied inversely with the timescale of the measurement.

To determine to what extent the variation we observed is a result of pooling together F-actin tracks from several different cells for analysis, we examined the velocity distributions for individual cells. We found some cellto-cell variability in velocity distributions outside stress fibers, and much less within stress fibers (Figure S5, A-B). Fits of the data from individual cells yielded fit parameters that were indistinguishable within error (see sections below), indicating that F-actin dynamics in individual cells were similar.

\section{F-actin velocity distribution fits can estimate physical parameters of underlying motion}

While the Weibull distribution yielded an excellent empirical description of our data, it provides limited physical insight into the mechanistic processes that drive F-actin motion. We therefore sought to develop a reductionist physical model of $\mathrm{F}$-actin motion that could potentially account for our observations. As with all reductionist models, it likely fails to capture important aspects of cellular behavior (here, F-actin dynamics) that arise from the highly complex molecular milieu of the cell. Moreover, as with all such models, its correctness cannot be "proven", but only invalidated by experimental data. Nevertheless, we view such models as useful in testing physical intuition, and in generating predictions that can potentially stimulate future experiments.

In our working model, we assume F-actin movement in the cell to arise from a combination of diffusive motion, bulk drift, and jump processes. We therefore interpreted our data by comparing two extremes: one in which $\mathrm{F}$-actin moves solely by diffusion with drift (Figure 4A), and one in which F-actin moves solely by a jump process (Figure 4C). For parsimony, we modeled each by its simplest two-parameter formulation. For a population which moves with some drift velocity in one direction and diffusion in two dimensions, the step size is described by a Rice distribution (identical to the non-central $\chi$ distribution with two degrees of freedom, but parameterized in a way that is more intuitive in this context). Statistical jump processes are less often thought about in a biological context, and describe a system where particles exist primarily in a 

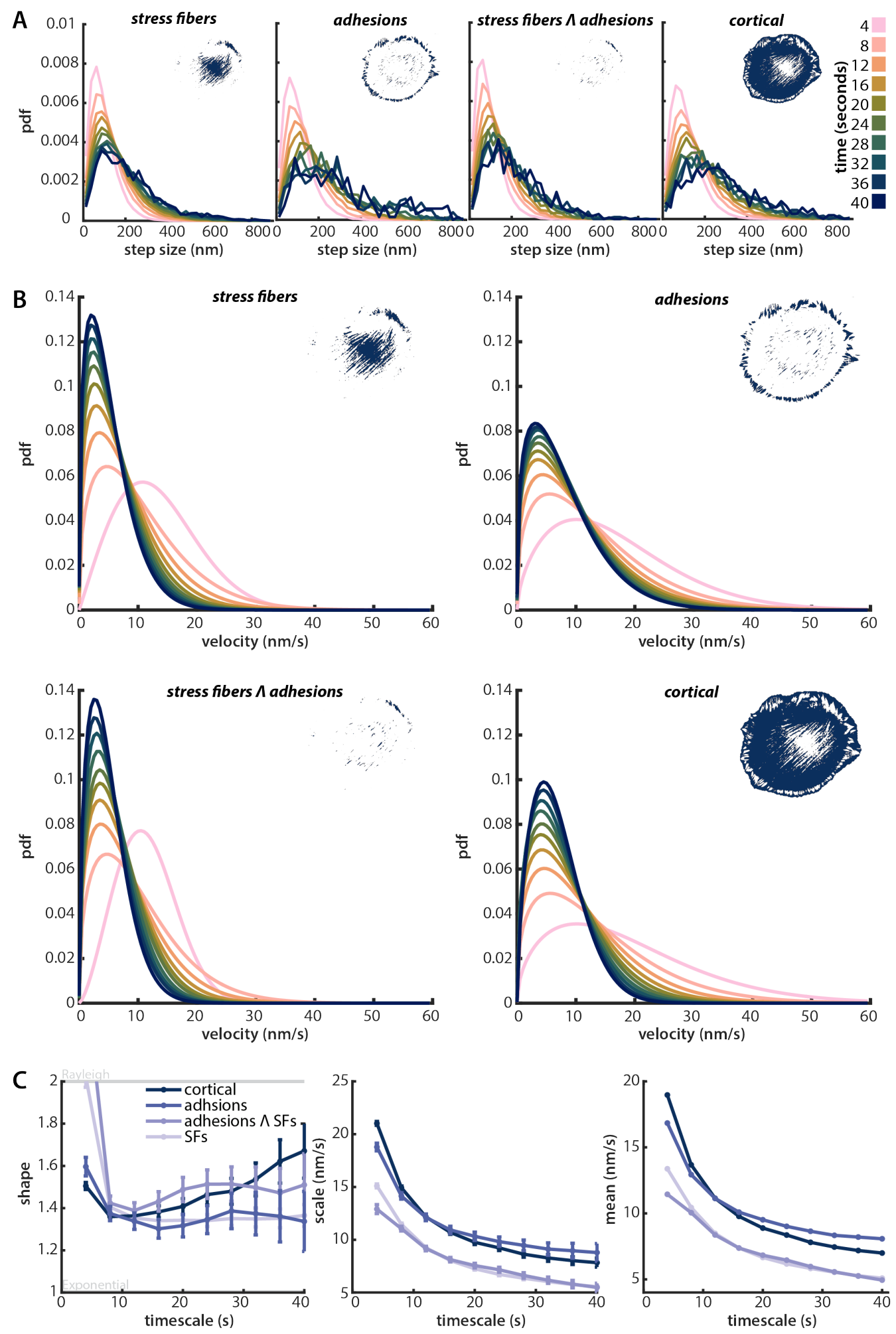

FIG. 3. Inferred distributions of F-actin velocities show distinct behavior inside vs. outside SFs. A) Puncta were automatically classified into four subpopulations. Displacements for F-actin puncta in the four subpopulations are shown for varying time intervals. These four regions are the four mututally-exclusive combinations of $(+/-)$ stress fibers and $(+/-$ ) adhesions: the cortical population is taken to be F-actin outside both stress fibers and adhesions. The distribution of displacements is calculated for timescales ranging from 2 seconds to 40 seconds, in 2 second intervals. Only every other time interval is shown for clarity. B) The inferred velocity distributions corresponding to the measured distance distributions in (A). Shaded regions represent $95 \%$ confidence intervals on the fits. C) Left, center: The fit parameters for the Weibull-distribution fits in (B). Error bars show the $95 \%$ confidence interval for each parameter. Right: the average velocity for each population and timescale, calculated from the best-fit Weibull distribution. 

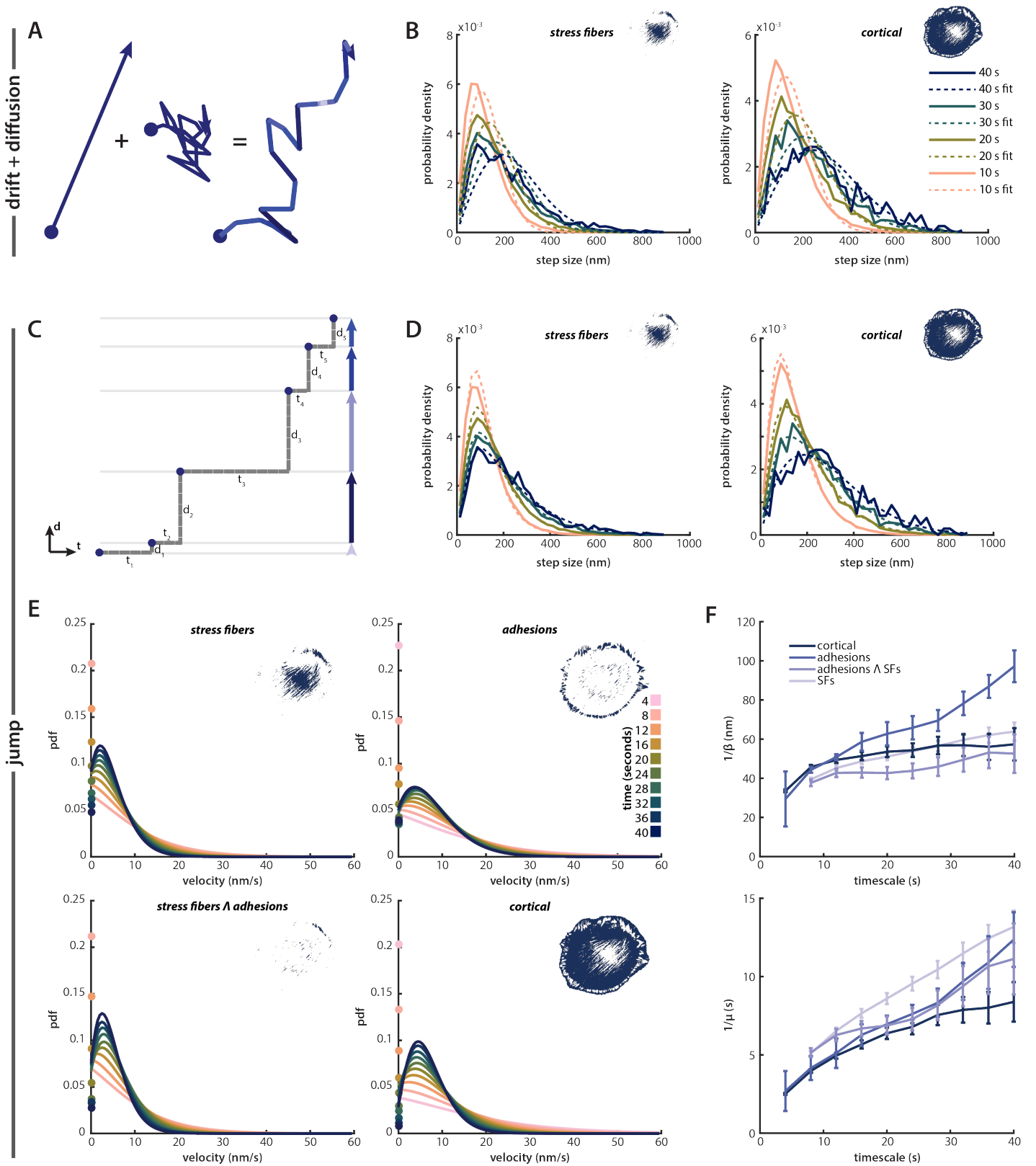

FIG. 4. F-actin velocity distributions in HFFs are well-fit by a jump process model with exponentially distributed waiting times and jump distances. A) Schematic of the diffusion with drift model. Persistent drift (left) is coupled with diffusion (center), leading to the motion shown on the right. The velocity of each step correlates with the darkness of the line (darker colors signify faster motion). B) The best fit of the diffusion with drift model (dashed lines) with the data (solid lines) for both cytosolic and stress fiber populations. The fits are shown for four timescales. C) Schematic of the jump model. The one-dimensional motion is shown both in two-dimensional distance-time space (left) and in one-dimensional space (right), where the arrow color again correlates with the average velocity (jump distance over wait time) of the segment. D) The best fit of the jump model (dashed lines) with the data (solid lines), as in B. E) The resulting velocity distributions for the best-fit jump model parameters, for all four populations, over timescales from 4 to $40 \mathrm{~s}$. The distribution is discontinuous at zero, and filled circles on the y-axis represent the discrete probability of a particle remaining motionless over the given observation time. F) The best fit parameters for the jump process, for all timescales measured. The characteristic time is given by $1 / \mu$ and the characteristic distance by $1 / \beta$. Error bars show $95 \%$ confidence intervals on the fit parameters.

stationary state interrupted by instantaneous, discrete jumps. While the motion of the F-actin cytoskeleton cannot be truly instantaneous, this provides a useful framework to approximate processes like the rapid slipping and sticking in the molecular clutch model[40], or those that might be expected from soft glass or gel descriptions of the cytoskeleton[26-28]. For the simplest jump motion process, we assume both the jump time and 
jump distance are exponentially distributed.

Surprisingly, the model of diffusion with drift did not fit the data well (Figure 4B), and yielded aphysical values for fit parameters. The best fit parameters for this model give a mean drift speed $\sim 0.01 \mathrm{~nm} / \mathrm{s}$ for all populations and a diffusive term of $\sim 700 \mathrm{~nm}^{2} / \mathrm{s}$ for stress fibers and $\sim 1200 \mathrm{~nm}^{2} / \mathrm{s}$ for the cortical population. These numbers imply that movement of filaments should be overwhelmingly dominated by diffusive, rather than drift, motion. This seems improbable given our observations, where individual fiducials appear to move in a persistent, directed fashion (Figure 1B).

In contrast, the jump process captures the data for all four populations well (Figure 4D and Figure S6). In this simple formulation, any piece of the cytoskeleton moves some random distance $s$ after waiting a random time $t$. At each time, a new distance and wait time are sampled. The distance $s$ and the time $t$ are both exponentially distributed, with means $1 / \beta$ and $1 / \mu$, respectively. Fits to this model gave mean switching distances and timescales of $\sim 29-97 \mathrm{~nm}$ and $\sim 2.5-13 \mathrm{~s}$, respectively (Figure 4F). We also tracked F-actin fiducials in human umbilical vein epithelial cells (HUVECs), which when plated on glass formed numerous, well aligned stress fibers. This F-actin in this cell type was best fit by a jump process with jump distances similar to HFFs, and somewhat longer waiting times (up to $\sim 20 \mathrm{~s}$ ) between jumps (Figure S7).

Interestingly, the characteristic distance was almost indistinguishable between the stress fiber and cortical populations in HFFs, while the characteristic timescale varied between these subpopulations, giving longer timescales $(\sim 5.1-13.2 \mathrm{~s})$ for the stress fiber population and shorter $(\sim 2.5-8.4 \mathrm{~s})$ for the cortical population. Adhesions that were not colocalized with stress fibers were best fit by longer jump distances than the other four populations. When step sizes from individual cells were fit to this distribution, there was some cell-to-cell variability, but there is a consistent trend, where the length scale in cortical and stress fiber populations are similar, while the time scale tends to be longer in stress fibers (Figure S5, C-D).

This model introduces a discontinuous peak at zero, given that there is finite probability that a particle does not jump at all during an observation time (Figure $4 \mathrm{E})$. The fraction of the population that is motionless is set only by the characteristic timescale of the jumps and the timescale of observation, and is independent of the characteristic jump length. This probability is high at short timescales (21\% for stress fibers and $13 \%$ for cortical at the $8 \mathrm{~s}$ timescale) and diminishes at longer timescales ( $5 \%$ for stress fibers and $0.8 \%$ for cortical at the $40 \mathrm{~s}$ timescale). While zero displacement is impossible to measure directly due to localization error, this recovered population from fitting is consistent with the observation that many puncta appeared to be stationary by eye or by kymograph, particularly in stress fibers.
Comparing the likelihood ratio of the best-fit jump process with the best-fit diffusive drift showed that the the jump process fits are always more likely. The fits from the two models are most comparable for the cortical population at long $(40 \mathrm{~s})$ timescales, where the jump process is still $10^{5.7}$ times more likely, while for stress fibers at shorter $(20 \mathrm{~s})$ timescales, the likelihood ratio is much larger $\left(10^{611}\right)$.

The fits to the jump process model give a somewhat different shape from the Weibull distribution fits, despite both fitting the data much better than the other distributions we tried (exponential, Gaussian, and Rayleigh). However, comparison of the likelihoods of both models showed the jump process to give higher likelihoods by factors of $10^{0.7}$ to $10^{225}$ for varying populations and timescales. This illustrates that multiple models can fit the data well, but that the use of maximum likelihood fitting and likelihood ratios allow us to leverage all collected data points in our fits to compare models.

\section{DISCUSSION}

We have described and applied a method to infer velocity distributions for slow single molecule tracks in living cells. While recent microscopy techniques have allowed precise measurements with single nanometer accuracy in vitro[41], these advances are often difficult to apply to commonly used in vivo fluorescent probes. Because our technique does not require high precision to reconstruct a population with slow-moving molecules, we anticipate that it may be useful in the analysis of other particle tracking measurements that face limitations imposed by low signal-to-noise ratios. The approach we outline here is also applicable to the statistical description of noisy distance measurements, for example in the context of two-color STORM/PALM microscopy experiments, and may thus be generally useful in interpreting superresolution data.

While a number of models can be fit to the data, we begin to eliminate some whose best fit parameters provide aphysical interpretations. For example, the best fit parameters from a model of diffusion and drift give drift speeds on the order of $0.1 \mathrm{~nm} / \mathrm{s}$ and diffusion on the order of $1000 \mathrm{~nm}^{2} / \mathrm{s}$. Goodness of fit also allowed us to eliminate other simple distributions (exponential, Gaussian, Rayleigh), which constrains possible models of actin motion and force transmission.

The jump process, which fit the data best amongst the models tested, describes a system with exponentiallydistributed jump distances and times. It was surprising that this model fits the data significantly better than the more common diffusion with drift framework. While we don't observe obvious jump-like movements in our movies, the average jump distance for most populations and timescales is between $40-65 \mathrm{~nm}$, close to our localization precision, and often less than our pixel size of $64 \mathrm{~nm}$. Given the exponential distribution of 
jump distances, the most common jump sizes would be expected to be close to zero, and a majority of jumps would be less than one pixel. Physically, Factin motion is unlikely to be truly instantaneous, as in the purest model of a jump process, but these fits may reflect jump-like movements that occur below the time resolution of our measurement. As in other studies, we noted fast, inward-directed fiducial motion at the cell's periphery, consistent with retrograde flow in lamellipodia, but these make up a small number of our tracks (Video S1). It is plausible that F-actin motion in these regions is dominated by advective flow, i.e. drift, with respect to the laboratory rest frame, as in previous studies that focused on lamellipodial Factin dynamics. Whether filaments additionally undergo jump-type motion within flowing lamellipodial/lamellar actin remains to be determined.

Although we emphasize the provisionality of the jump model, it is interesting to consider its physical interpretation. The good fit of the jump process relative to that of diffusion and drift, may imply that these Factin structures are more elastic than viscous on these timescales (10 s - $2 \mathrm{~min})$. This picture is consistent with previous microrheology measurements, which found that the cell cytoplasm as a whole is predominantly elastic on sub-second to few seconds timescales[26, 27, 29]. One interpretation of the model is that, on this timescale, F-actin exists in a crosslinked network that is close to mechanical equilibrium. Disruption of this equilibrium by either crosslinkers unbinding or force generation by myosin occurs in discrete jumps reflecting the intrinsically quantized, molecular nature of these processes. In this interpretation, the characteristic lengths and waiting times of the actin cortex and stress fibers reflect the emergent properties of each structure. For example, the characteristic length may be set by the density of actin crosslinkers and binding proteins. In this regard it is intriguing that the magnitude of $1 / \beta$ $\sim 50 \mathrm{~nm}$ is similar to the $36 \mathrm{~nm}$ F-actin pseudohelical repeat. Waiting times that span roughly 5 to $10 \mathrm{~s}$ are commensurate with the timescale of F-actin crosslinker rearrangement inferred in a recent study from our laboratory[34].

It is perhaps surprising that the jump model, or any model, fits the data given that the the datasets combine single-molecule tracks from multiple subcellular regions, and for that matter multiple cells. We speculate that the values of $\mu$ and $\beta$ emerge from the broad distributions of timescales (and possibly length scales) that might be expected from the myriad of actin-binding proteins with varied kinetics. Whether these emergent parameters reflect a trivial consequence of the central limit theorem or instead have a more complex physical origin remains to be determined.

The fit of the jump model to the data revealed that the time constant $(1 / \mu)$ increased roughly linearly with the time delay between measurements (Fig. 4F). The reason(s) for this time dependence are unclear. We note however that the timescale of our measurement is commensurate with the rate of F-actin turnover in the cell. One possibility is thus that different F-actin populations possess different dynamics: measurements at longer timescales (e.g. $40 \mathrm{~s}$ ) are likely enriched for longer, more stable filaments. Future explorations that quantify F-actin dynamics on shorter $(0.1-1 \mathrm{~s})$ and longer (10-100 s) may shed light on this interesting question.

In summary, we find that a simple jump model is sufficient to describe the motion of four cellular F-actin populations in the cell types examined here. While any number of models may also account for our observations, this model is statistically the most likely of those we have tested. Future work can help to elucidate the underlying molecular mechanisms that set the observed velocity distributions and more particularly, the biophysical origins of the distances $(1 / \beta)$ and timescales $(1 / m u)$ implied by the jump model. It will likewise be of interest to determine how these properties vary across cell types, lending insight into how cells tune the molecular-scale dynamics of the cytoskeleton to fulfill different biological functions.

\section{MATERIALS AND METHODS}

\section{A. Cell culture}

HFF cells CCD-1070Sk (ATCC CRL-2091) were cultured in DMEM high-glucose medium (Gibco, catalog no. 21063-029) in the absence of phenol red and supplemented with $10 \%$ fetal bovine serum (Corning 35-011-CV), sodium pyruvate (1 mM, Gibco 11360070), MEM nonessential amino acids (1x; Gibco 11140050), and penicillin/streptomycin (100 U/ml; Gibco 15140122). Fibroblasts stably expressing eGFPpaxillin (fused at the C-terminus) were prepared as previously described[42]. HUVEC cells (Lonza C2591A) were cultured in EGM-2 basal medium (Lonza CC-3156) with the addition of EGM-2 MV Bullet Kit (Lonza CC-4147), with the exception of gentamicin. Instead, penicillin-streptomycin (Gibco 15140122) is added to a concentration of $100 \mathrm{U} / \mathrm{ml}$. All cells were grown at 37 ${ }^{\circ} \mathrm{C}$ with $5 \% \mathrm{CO} 2$.

\section{B. Experimental setup and imaging}

Halo-PEG coverslips were prepared as previously[34]. Coverslips with coverwell chambers were functionalized with an RGD-presenting protein force sensor, here without fluorescent dyes, as described previously[34]. In brief, Halo-ligand functionalized coverslips were incubated at room temperature for $30 \mathrm{~min}$ with 100 $\mathrm{nM}$ unlabelled RGD-presenting sensor, which contains a HaloTag for attachment. The coverslip chambers were then rinsed with PBS before cells were seeded and allowed to spread for at least $1 \mathrm{hr}$. After a 9 minute 
incubation with $50 \mathrm{nM}$ SiR-Actin (Cytoskeleton Inc., CY-SC001), the sample was incubated with Prolong Live Antifade Reagent (Invitrogen P36975) for $1 \mathrm{hr}$. The low dilution of SiR-Actin allowed for individual molecules to be tracked, and the addition of Prolong reduced photobleaching. For each cell, a $100 \mathrm{~ms}$ exposure of the GFP-paxillin channel (for masking) was first acquired, followed by a 60 frame sequence in the far red channel (SiR-Actin) with $300 \mathrm{~ms}$ exposures taken every 2 seconds. For fixed cell data, cells were allowed to spread on functionalized coverslips and then fixed in $4 \%$ paraformaldehyde for 15 minutes at room temperature. After rinsing, the cells were treated with SiR-Actin and Prolong reagent as above.

\section{Actin tracking analysis}

Speckles and tracks were identified using QFSM software made available by the Danuser lab[39] and analyzed using the workflow in Fig. 2G. The localizations, which were given with pixel precision by QFSM, were fit to subpixel positions by Gaussian fitting. Drift was calculated for each movie using the drift estimation module of NanoJ[43]. The calculated drift values in $\mathrm{x}$ and $\mathrm{y}$ were then subtracted from the $\mathrm{x}$ and $\mathrm{y}$ positions of each point in the track before calculating frame-to-frame distances. Tracks were sorted into subcellular populations according to the location of the first point of the track. The total cell area was masked using a mean threshold of the GFP-paxillin image, where cytoplasmic paxillin signal was used to segment the cell. After dilating and eroding to fill small holes, the edge of this mask was smoothed by fitting to a cubic spline curve. Adhesions were masked by an Otsu threshold of the same GFP-paxillin image after background subtraction to remove the diffuse cytoplasmic signal. Actin stress fibers were masked as the brightest $2 \%$ of pixels of a time-series projection of the actin tracks. Finally, the cytosol was taken as regions within the cell mask which are excluded from both the actin mask and the paxillin mask.

To derive an empirical estimate of the localization error, we acquired SiR-actin tracks in paraformaldehyde fixed cells exactly as described above and used the displacements of these tracks to determine the measurement noise. The displacements of these fixed cell tracks were fit to the noncentral $\chi$ distribution (described in Appendix A) with $s=0$ by MLE.

Frame-to-frame displacements were calculated for varying frame intervals, from 1 frame $(2 \mathrm{~s})$ to 20 frames (40 s). The displacement distribution for each interval was first fit by least-squares to quickly estimate the best fit parameters, and these parameters were used to seed the MLE fit to equation 1. The resulting distributions were rescaled by the time interval to give velocity distributions. At the shortest timescales, the distributions were almost indistinguishable from the fixed cell distribution, and the MLE fits sometimes failed to converge (Figure S6), and so we focused on the longer timescales $(\geq 8 \mathrm{~s})$ in interpreting our results.

\section{ACKNOWLEDGMENTS}

We thank S. Tan for providing RGD-presenting tension sensor and GFP-paxillin-expressing HFFs, C. Vasquez for assistance and advice with HUVEC handling, and V. Vachharajani, L. Owen, S. Tan, C. Hueschen, and B. Zhong for many useful discussions and feedback.

Funding: Research reported in this publication was supported by grants R01-CA172986 and U54-CA210190 to D.J.O. and R35-GM130332 to A.R.D. from the National Institutes of Health (NIH). The research of A.R.D. was supported, in part, by a Faculty Scholar from the Howard Hughes Medical Institute. C.M.M. was supported by Graduate Research Fellowships from the National Science Foundation (00039202) and the Stanford EDGE fellowship. E.K. acknowledges support from the Stanford Bio-X Fellowship. The contents of this publication are solely the responsibility of the authors and do not necessarily represent the official views of the NIH.

\section{Appendix A: Derivation of distance distribution}

The analytical form for the distribution of measured distances, $d$, given a true distance, $s$, is an extension of the derivation by Churchmann et al.[44].

If we assume that two points are located at $(0,0)$ and $(s, 0)$, and that the measured coordinates are Gaussian distributed around the true location with variance $\sigma^{2}$, we can say that $x_{1}, y_{1}, y_{2} \sim N\left(0, \sigma^{2}\right)$ and $x_{2} \sim N\left(s, \sigma^{2}\right)$. As a result, the measured distance along $\mathrm{x}$ and $\mathrm{y}, d_{x}$ and $d_{y}$, respectively, are also normally distributed.

$$
\begin{aligned}
& d_{x}=x_{2}-x_{1} \sim N\left(s, 2 \sigma^{2}\right) \\
& d_{y}=y_{2}-y_{1} \sim N\left(0,2 \sigma^{2}\right)
\end{aligned}
$$

The noncentral $\chi$ distribution describes the distribution of a random variable $z$ given by

$$
z=\sqrt{\sum_{i}^{k}\left(\frac{X_{i}}{\sigma_{i}}\right)^{2}}
$$

where $X_{i}$ are normally distributed random variables with means $\mu_{i}$ and variances $\sigma_{i}^{2}$.

Therefore we may define $z$ in terms of $d$ as a noncentral $\chi$-distributed random variable with $k=2$ :

$$
z=\sqrt{\left(\frac{d_{x}}{\sigma_{d x}}\right)^{2}+\left(\frac{d_{y}}{\sigma_{d y}}\right)^{2}}=\frac{d}{\sqrt{2} \sigma}
$$


Assuming $\sigma_{d x}$ and $\sigma_{d y}$ are equal, the noncentral $\chi$ distribution is

$f_{z}(z, k, \lambda)=\lambda(\lambda z)^{-k / 2} \exp \left(-\frac{z^{2}+\lambda^{2}}{2}\right)\left(z^{k}\right) I_{k / 2-1}(\lambda z)$

where $I_{k / 2-1}$ is a modified Bessel function of the first kind and

$$
\lambda=\sqrt{\sum_{i}^{k}\left(\frac{\mu_{i}}{\sigma_{i}}\right)^{2}}=\sqrt{\frac{s^{2}}{2 \sigma^{2}}+\frac{0^{2}}{2 \sigma^{2}}}=\frac{s}{\sqrt{2} \sigma} .
$$

Scaling by $\sqrt{2} \sigma$ to determine the distribution of $d$, and substituting $k=2$ and $\lambda=s \sigma^{-1} / \sqrt{2}$, we find that

$$
f_{d}(d, s, \sigma)=\frac{d}{2 \sigma^{2}} \exp \left(-\frac{1}{4 \sigma^{2}}\left(d^{2}+s^{2}\right)\right) I_{0}\left(\frac{d s}{2 \sigma^{2}}\right)
$$

\section{Appendix B: Drift-diffusion and jump process models}

We modeled F-actin movement with two simple driftdiffusion models that we will collectively call the driftdiffusion model. In the first model, the particles undergo 1-D Brownian motion while drifting at a constant 1D velocity. This results in folded Gaussian distributed distances, with location parameter $\mu$ equal to the drift velocity times the observation time and spread parameter $\sigma^{2}$ equal to the diffusion coefficient times observation time. The second model differs from the first only in that, the particles are allowed to diffuse in 2-D while drifting in 1-D, which results in Rice distributed distances with center $\nu$ equal to the drift velocity times the observation time and spread parameter $\sigma^{2}$ equal to the diffusion coefficient times observation time.

We also modeled F-actin movement with a simple one dimensional unidirectional jump process with independent identically distributed (IID) exponential wait times between jumps and IID exponential jump distances at jumps. This function is sufficiently simple that we can obtain the solution in real space. When observed at a time interval $\tau$, the total number of jumps in the interval will be Poisson distributed with mean $\mu \tau$ where $\mu$ is the jump rate.

If $k$ non-zero jumps occur in an interval, the total distance moved in that interval will be distributed as the sum of $k$ IID exponential random variables each with some rate parameter $\beta$. This gives a Gamma distribution of distances with shape parameter $k$ and rate parameter $\beta$.

To find the probability distribution of the distance $x$ moved for a given observation time window $\tau$, we first need to sum up the probability that there is one jump of size $x$; two jumps of total size $x$; three jumps of total size $x$; and so on, giving

$$
\begin{array}{r}
\sum_{k=1}^{\infty} \frac{(\mu \tau)^{k}}{k !} e^{-\mu \tau} \gamma(k, \beta) \\
=\sum_{k=1}^{\infty} \frac{(\mu \tau)^{k}}{k !} e^{-\mu \tau} e^{-\beta x} x^{k-1} \frac{\beta^{k}}{(k-1) !} \\
=\frac{1}{x} e^{-\mu \tau-\beta x} \sum_{k=1}^{\infty} \frac{(\mu \tau \beta x)^{k}}{k !(k-1) !} \\
=\frac{1}{x} e^{-\mu \tau-\beta x} \sqrt{\mu \tau \beta x} I_{1}(2 \sqrt{\mu \tau \beta x}) \\
=e^{-\mu \tau-\beta x} \sqrt{\frac{\mu \tau \beta}{x}} I_{1}(2 \sqrt{\mu \tau \beta x})
\end{array}
$$

where $\gamma(k, \beta)$ is the Gamma distribution with shape parameter $k$, rate parameter $\beta$ and $I_{1}$ is the modified Bessel function of the first kind, of order 1 . This expression is incomplete since we also need to consider the (discrete) probability of moving a distance of exactly $\mathrm{x}=0$, corresponding to the case of no jumps. The probability of a point moving exactly zero distance is equal to the probability that a jump does not occur in the given observation interval, such that $P(x=0)=$ $e^{-\mu \tau}$. Therefore, the complete normalized probability distribution for moving a distance $x \geq 0$ is defined piecewise as

$$
P(x)=\left\{\begin{array}{l}
e^{-\mu \tau} \text { for } x=0 \\
e^{-\mu \tau-\beta x} \sqrt{\frac{\mu \tau \beta}{x}} I_{1}(2 \sqrt{\mu \tau \beta x}) \text { for } x>0
\end{array}\right.
$$

\section{Appendix C: Numerical approximation of Bayes Theorem (Eqn. 1)}

The probability of observing a step distance, $d$, given a distribution of true distances, $f_{s}(s)$, is given by

$$
f_{d}(d)=\int_{-\infty}^{\infty} f_{d}(d \mid s=S) f_{s}(S) d S
$$

While this equation may be expressed analytically for some forms of $f_{s}(s)$, we fit to a numerical approximation of this equation for consistency and ease of application to various functional forms. For our case, where we fit to distances, no values are less than zero, and the limits become zero to infinity. We approximate this integral as a Riemann sum over the interval from 0 to $1000 \mathrm{~nm}$, with 100 linearly-spaced bin edges (bin width $=10 \mathrm{~nm}$ ). For each bin, both $f_{s}(S)$ and $f_{d}(d \mid s=S)$ are calculated at both bin edges, averaged, and multiplied by the bin width. These are summed to give $f_{d}(d)$.

For the jump process as described in Appendix B, Bayes rule is not defined exactly as above due to the discrete discontinuity at $x=0$. Instead, because 
the probability is defined piece-wise (Eqn. B2), Bayes theorem is rewritten in this case to give

$f_{d}(d)=f_{d}(d \mid s=0) f_{s}(s=0)+\int_{0+}^{\infty} f_{d}(d \mid s=S) f_{s}(S) d S$
We approximate the integral here by approaching zero from the right, using 100 linearly-spaced bin edges from 0.0001 to $1000 \mathrm{~nm}$.
[1] J. V. Small, K. Rottner, I. Kaverina, and K. Anderson, Assembling an actin cytoskeleton for cell attachment and movement, Biochimica Et Biophysica Acta (BBA)Molecular Cell Research 1404, 271 (1998).

[2] A. Ponti, M. Machacek, S. Gupton, C. Waterman-Storer, and G. Danuser, Two distinct actin networks drive the protrusion of migrating cells, Science 305, 1782 (2004).

[3] D. T. Burnette, S. Manley, P. Sengupta, R. Sougrat, M. W. Davidson, B. Kachar, and J. Lippincott-Schwartz, A role for actin arcs in the leading-edge advance of migrating cells, Nature cell biology 13, 371 (2011).

[4] T. M. Svitkina, Ultrastructure of the actin cytoskeleton, Current opinion in cell biology 54, 1 (2018).

[5] M. Bovellan, Y. Romeo, M. Biro, A. Boden, P. Chugh, A. Yonis, M. Vaghela, M. Fritzsche, D. Moulding, R. Thorogate, et al., Cellular control of cortical actin nucleation, Current Biology 24, 1628 (2014).

[6] J. R. Soiné, C. A. Brand, J. Stricker, P. W. Oakes, M. L. Gardel, and U. S. Schwarz, Model-based traction force microscopy reveals differential tension in cellular actin bundles, PLoS Comput Biol 11, e1004076 (2015).

[7] K. Rottner, J. Faix, S. Bogdan, S. Linder, and E. Kerkhoff, Actin assembly mechanisms at a glance, J Cell Sci 130, 3427 (2017).

[8] Y. Cai, N. Biais, G. Giannone, M. Tanase, G. Jiang, J. M. Hofman, C. H. Wiggins, P. Silberzan, A. Buguin, B. Ladoux, and M. P. Sheetz, Nonmuscle myosin iiadependent force inhibits cell spreading and drives f-actin flow, Biophysical journal 91, 3907 (2006).

[9] J. A. Theriot and T. J. Mitchison, Actin microfilament dynamics in locomoting cells, Nature 352, 126 (1991).

[10] J. A. Theriot and T. J. Mitchison, Comparison of actin and cell surface dynamics in motile fibroblasts., The Journal of cell biology 119, 367 (1992).

[11] G. Giannone, B. J. Dubin-Thaler, H.-G. Döbereiner, N. Kieffer, A. R. Bresnick, and M. P. Sheetz, Periodic lamellipodial contractions correlate with rearward actin waves, Cell 116, 431 (2004).

[12] G. Giannone, B. J. Dubin-Thaler, O. Rossier, Y. Cai, O. Chaga, G. Jiang, W. Beaver, H.-G. Döbereiner, Y. Freund, G. Borisy, et al., Lamellipodial actin mechanically links myosin activity with adhesion-site formation, Cell 128, 561 (2007).

[13] L. Ji and G. Danuser, Tracking quasi-stationary flow of weak fluorescent signals by adaptive multi-frame correlation, Journal of microscopy 220, 150 (2005).

[14] A. Ponti, A. Matov, M. Adams, S. Gupton, C. Waterman-Storer, and G. Danuser, Periodic patterns of actin turnover in lamellipodia and lamellae of migrating epithelial cells analyzed by quantitative fluorescent speckle microscopy, Biophysical journal 89, 3456 (2005).
[15] P. Vallotton, S. L. Gupton, C. M. Waterman-Storer, and G. Danuser, Simultaneous mapping of filamentous actin flow and turnover in migrating cells by quantitative fluorescent speckle microscopy, Proceedings of the National Academy of Sciences 101, 9660 (2004).

[16] C. Jurado, J. R. Haserick, and J. Lee, Slipping or gripping? fluorescent speckle microscopy in fish keratocytes reveals two different mechanisms for generating a retrograde flow of actin, Molecular biology of the cell 16, 507 (2005).

[17] K. Hu, L. Ji, K. T. Applegate, G. Danuser, and C. M. Waterman-Storer, Differential transmission of actin motion within focal adhesions, Science 315, 111 (2007).

[18] M. L. Gardel, B. Sabass, L. Ji, G. Danuser, U. S. Schwarz, and C. M. Waterman, Traction stress in focal adhesions correlates biphasically with actin retrograde flow speed, The Journal of cell biology 183, 999 (2008).

[19] A. Y. Alexandrova, K. Arnold, S. Schaub, J. M. Vasiliev, J.-J. Meister, A. D. Bershadsky, and A. B. Verkhovsky, Comparative dynamics of retrograde actin flow and focal adhesions: formation of nascent adhesions triggers transition from fast to slow flow, PloS one 3 (2008).

[20] S. Yamashiro, H. Mizuno, M. B. Smith, G. L. Ryan, T. Kiuchi, D. Vavylonis, and N. Watanabe, New singlemolecule speckle microscopy reveals modification of the retrograde actin flow by focal adhesions at nanometer scales, Molecular biology of the cell 25, 1010 (2014).

[21] C. M. Brown, B. Hebert, D. L. Kolin, J. Zareno, L. Whitmore, A. R. Horwitz, and P. W. Wiseman, Probing the integrin-actin linkage using high-resolution protein velocity mapping, Journal of cell science 119, 5204 (2006).

[22] L. B. Case and C. M. Waterman, Integration of actin dynamics and cell adhesion by a three-dimensional, mechanosensitive molecular clutch, Nature cell biology 17, 955 (2015).

[23] L. M. Owen, A. S. Adhikari, M. Patel, P. Grimmer, N. Leijnse, M. C. Kim, J. Notbohm, C. Franck, and A. R. Dunn, A cytoskeletal clutch mediates cellular force transmission in a soft, three-dimensional extracellular matrix, Molecular biology of the cell 28, 1959 (2017).

[24] A. Ponti, P. Vallotton, W. Salmon, C. Waterman-Storer, and G. Danuser, Computational analysis of f-actin turnover in cortical actin meshworks using fluorescent speckle microscopy, Biophysical journal 84, 3336 (2003).

[25] B. Hebert, S. Costantino, and P. W. Wiseman, Spatiotemporal image correlation spectroscopy (stics) theory, verification, and application to protein velocity mapping in living cho cells, Biophysical journal 88, 3601 (2005).

[26] B. Fabry, G. N. Maksym, J. P. Butler, M. Glogauer, 
D. Navajas, and J. J. Fredberg, Scaling the microrheology of living cells, Physical review letters 87, 148102 (2001).

[27] P. Bursac, G. Lenormand, B. Fabry, M. Oliver, D. A. Weitz, V. Viasnoff, J. P. Butler, and J. J. Fredberg, Cytoskeletal remodelling and slow dynamics in the living cell, Nature materials 4, 557 (2005).

[28] K. K. Mandadapu, S. Govindjee, and M. R. Mofrad, On the cytoskeleton and soft glassy rheology, Journal of biomechanics 41, 1467 (2008).

[29] M. Guo, A. J. Ehrlicher, M. H. Jensen, M. Renz, J. R. Moore, R. D. Goldman, J. Lippincott-Schwartz, F. C. Mackintosh, and D. A. Weitz, Probing the stochastic, motor-driven properties of the cytoplasm using force spectrum microscopy, Cell 158, 822 (2014).

[30] J. Fredberg and B. Fabry, The cytoskeleton as a soft glassy material, in Cytoskeletal Mechanics: Models and Measurements in Cell Mechanics, Cambridge Texts in Biomedical Engineering, edited by M. R. K. Mofrad and R. D. Kamm (Cambridge University Press, 2006) p. $50-70$.

[31] G. Danuser and C. M. Waterman-Storer, Quantitative fluorescent speckle microscopy of cytoskeleton dynamics, Annual review of biophysics and biomolecular structure 35 (2006).

[32] N. Watanabe and T. J. Mitchison, Single-molecule speckle analysis of actin filament turnover in lamellipodia, Science 295, 1083 (2002).

[33] S. Yamashiro, S. Tanaka, L. M. McMillen, D. Taniguchi, D. Vavylonis, and N. Watanabe, Myosin-dependent actin stabilization as revealed by single-molecule imaging of actin turnover, Molecular biology of the cell 29, 1941 (2018).

[34] S. J. Tan, A. C. Chang, S. M. Anderson, C. M. Miller, L. S. Prahl, D. J. Odde, and A. R. Dunn, Regulation and dynamics of force transmission at individual cell-matrix adhesion bonds, Science Advances 6, eaax0317 (2020).

[35] E. Zaccarelli, Colloidal gels: equilibrium and non- equilibrium routes, Journal of Physics: Condensed Matter 19, 323101 (2007).

[36] M. Melak, M. Plessner, and R. Grosse, Actin visualization at a glance, J Cell Sci 130, 525 (2017).

[37] T. Vignaud, C. Copos, C. Leterrier, Q. Tseng, L. Blanchoin, A. Mogilner, M. Théry, and L. Kurzawa, Stress fibers are embedded in a contractile cortical network, bioRxiv (2020).

[38] J. A. Waddle, T. S. Karpova, R. H. Waterston, and J. A. Cooper, Movement of cortical actin patches in yeast., The Journal of cell biology 132, 861 (1996).

[39] M. C. Mendoza, S. Besson, and G. Danuser, Quantitative fluorescent speckle microscopy (qfsm) to measure actin dynamics, Current protocols in cytometry 62, 2 (2012).

[40] T. Mitchison and M. Kirschner, Cytoskeletal dynamics and nerve growth, Neuron 1, 761 (1988).

[41] S. Niekamp, J. Sung, W. Huynh, G. Bhabha, R. D. Vale, and N. Stuurman, Nanometer-accuracy distance measurements between fluorophores at the singlemolecule level, Proceedings of the National Academy of Sciences 116, 4275 (2019).

[42] M. Morimatsu, A. H. Mekhdjian, A. C. Chang, S. J. Tan, and A. R. Dunn, Visualizing the interior architecture of focal adhesions with high-resolution traction maps, Nano letters 15, 2220 (2015).

[43] R. F. Laine, K. L. Tosheva, N. Gustafsson, R. D. Gray, P. Almada, D. Albrecht, G. T. Risa, F. Hurtig, A.-C. Lindås, B. Baum, et al., Nanoj: a highperformance open-source super-resolution microscopy toolbox, Journal of Physics D: Applied Physics 52, 163001 (2019).

[44] L. S. Churchman, Z. Ökten, R. S. Rock, J. F. Dawson, and J. A. Spudich, Single molecule highresolution colocalization of cy3 and cy5 attached to macromolecules measures intramolecular distances through time, Proceedings of the National Academy of Sciences 102, 1419 (2005). 
bioRxiv preprint doi: https://doi.org/10.1101/2020.08.13.247304; this version posted August 14,2020 . The copyright holder for this preprint (which was not certified by peer review) is the author/funder, who has granted bioRxiv a license to display the preprint in perpetuity. It is made available under aCC-BY-NC-ND 4.0 International license.

TABLE S1. Log10(likelihood-ratios) for several two-parameter model comparisons, abbreviated as follows: Weibull: Wbl, folded Gaussian: Fgaus.

\begin{tabular}{ccccccccc} 
Population & Wbl vs. Fgaus & \multicolumn{2}{c}{ Jump vs. Rice } & \multicolumn{2}{c}{ Jump vs. Wbl } & \multicolumn{2}{c}{ Jump vs. Fgaus } \\
\hline timescale & $20 \mathrm{~s}$ & $40 \mathrm{~s}$ & $20 \mathrm{~s}$ & $40 \mathrm{~s}$ & $20 \mathrm{~s}$ & $40 \mathrm{~s}$ & $20 \mathrm{~s}$ & $40 \mathrm{~s}$ \\
\hline SFs & 3238 & 2037 & 611 & 113 & 225 & 19 & 3464 & 2057 \\
cortical & 1871 & 217 & 204 & 5.7 & 45 & 0.96 & 1916 & 218 \\
adhesions & 774 & 235 & 117 & 15 & 16 & 0.69 & 790 & 236 \\
adhesions $\wedge$ SFs & 820 & 117 & 85 & 9.8 & 39 & 2.5 & 859 & 120 \\
\hline
\end{tabular}


bioRxiv preprint dol: https://doi.org/10.1101/2020.08.13.247304; this version posted August 14, 2020. The copyright holder for this preprint

(which was not certified by peer review) is the author/funder, who has granted bioRxiv a license to display the preprint in perpetuity. It is made available under aCC-BY-NC-ND 4.0 International license.

15

A
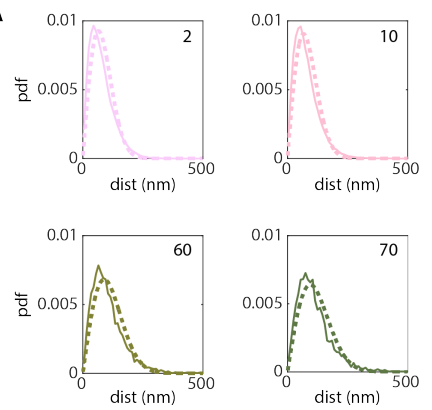
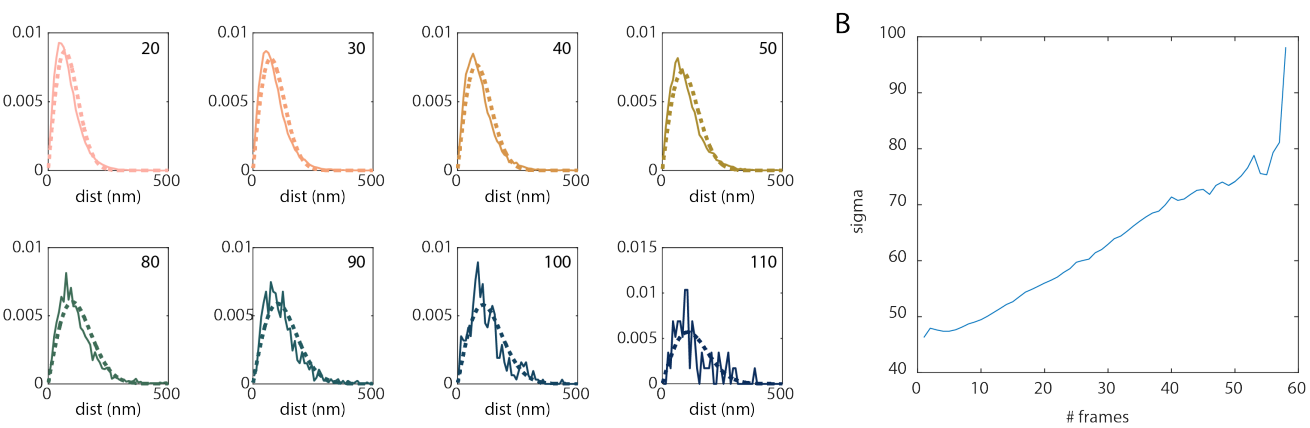

FIG. S1. A. Fixed cell distributions for HFFs, with varying number of frames, each $2 \mathrm{~s}$ apart (time interval, in seconds, indicated in the top right corner of each). The best fit distribution to the noncentral-chi description with $\mathrm{s}=0$ and varying $\sigma$ is shown in dashed lines. B. The best fit $\sigma$ for each timescale. The fit by the noncentral chi for data acquired with 2 to $10 \mathrm{~s}$ time delays gives approximately the same localization error $(47 \mathrm{~nm})$ before beginning to rise at longer timescales (up to $56 \mathrm{~nm}$ over $40 \mathrm{~s}$ ). We take the former as the best estimate of our localization error, as it represents our most static sample, and use this in our downstream workflow. 
bioRxiv preprint doi: https://doi.org/10.1101/2020.08.13.247304; this version posted August 14,2020 . The copyright holder for this preprint (which was not certified by peer review) is the author/funder, who has granted bioRxiv a license to display the preprint in perpetuity. It is made available under aCC-BY-NC-ND 4.0 International license.

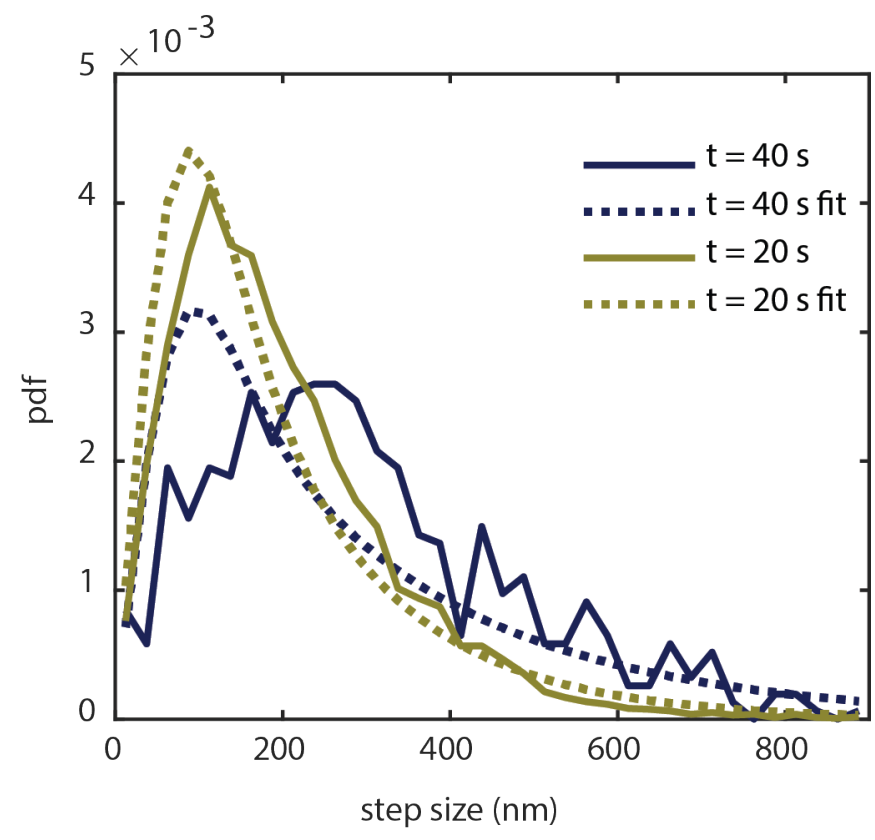

FIG. S2. Exponential fits to the cortical population in HFFs, at the $20 \mathrm{~s}$ and $40 \mathrm{~s}$ timescales, show poor fits to the data. 

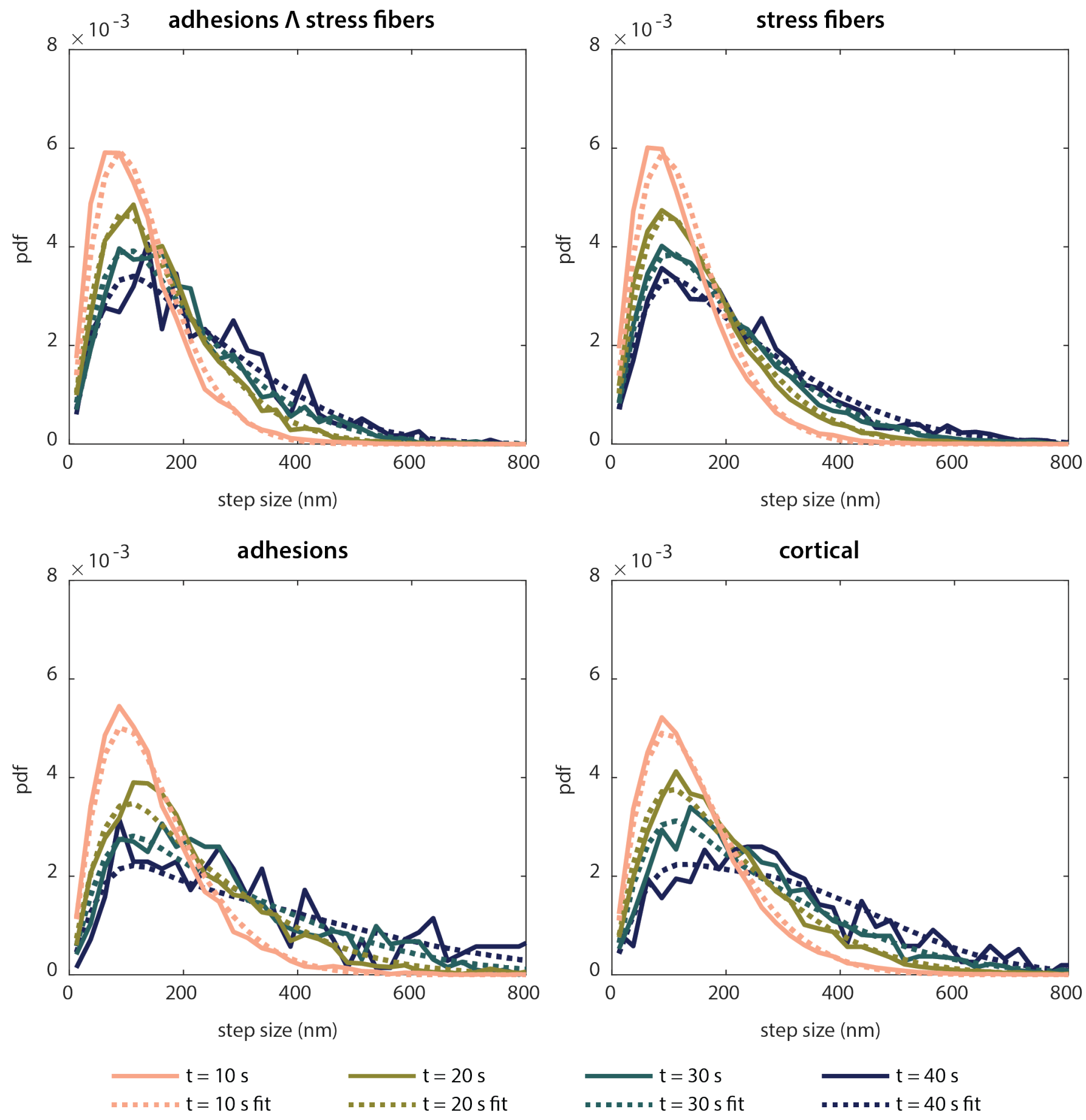

FIG. S3. Folded Gaussian fits to all four populations over varying timescales (10, 20, 30, and 40 s). The folded Gaussian is less heavy-tailed than the measured distribution, and tends to either underestimate the tails (most noticeable at the $10 \mathrm{~s}$ timescale on a semilog plot) or miss the peak in order to fit the tails (e.g. the adhesion and cortical fits at longer timescales). 

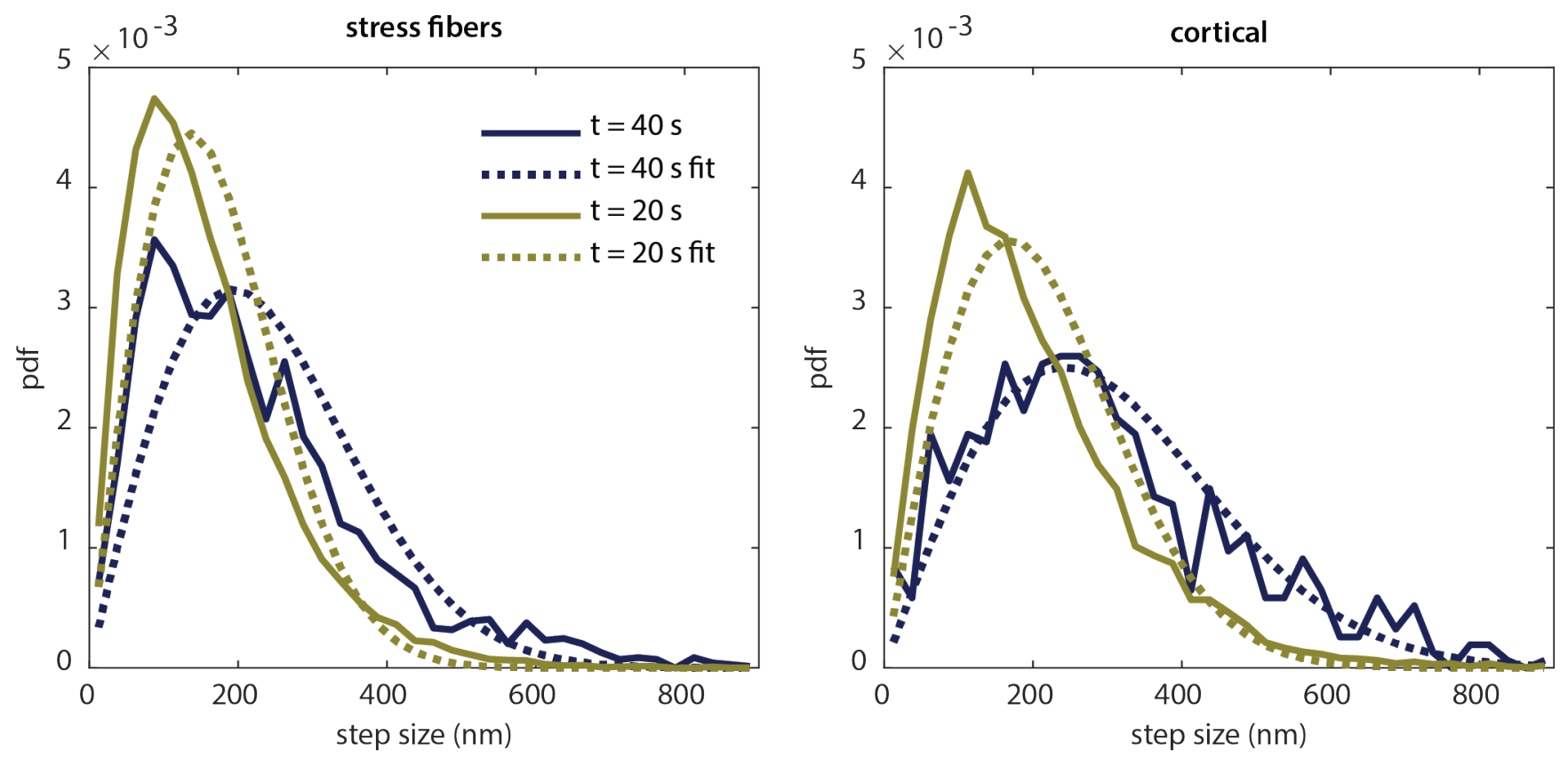

FIG. S4. Rayleigh fits to both stress fiber and cortical populations in HFFs, at the $20 \mathrm{~s}$ and $40 \mathrm{~s}$ timescales, showing that only the cortical population at the longest timescale is somewhat well-fit. 
A
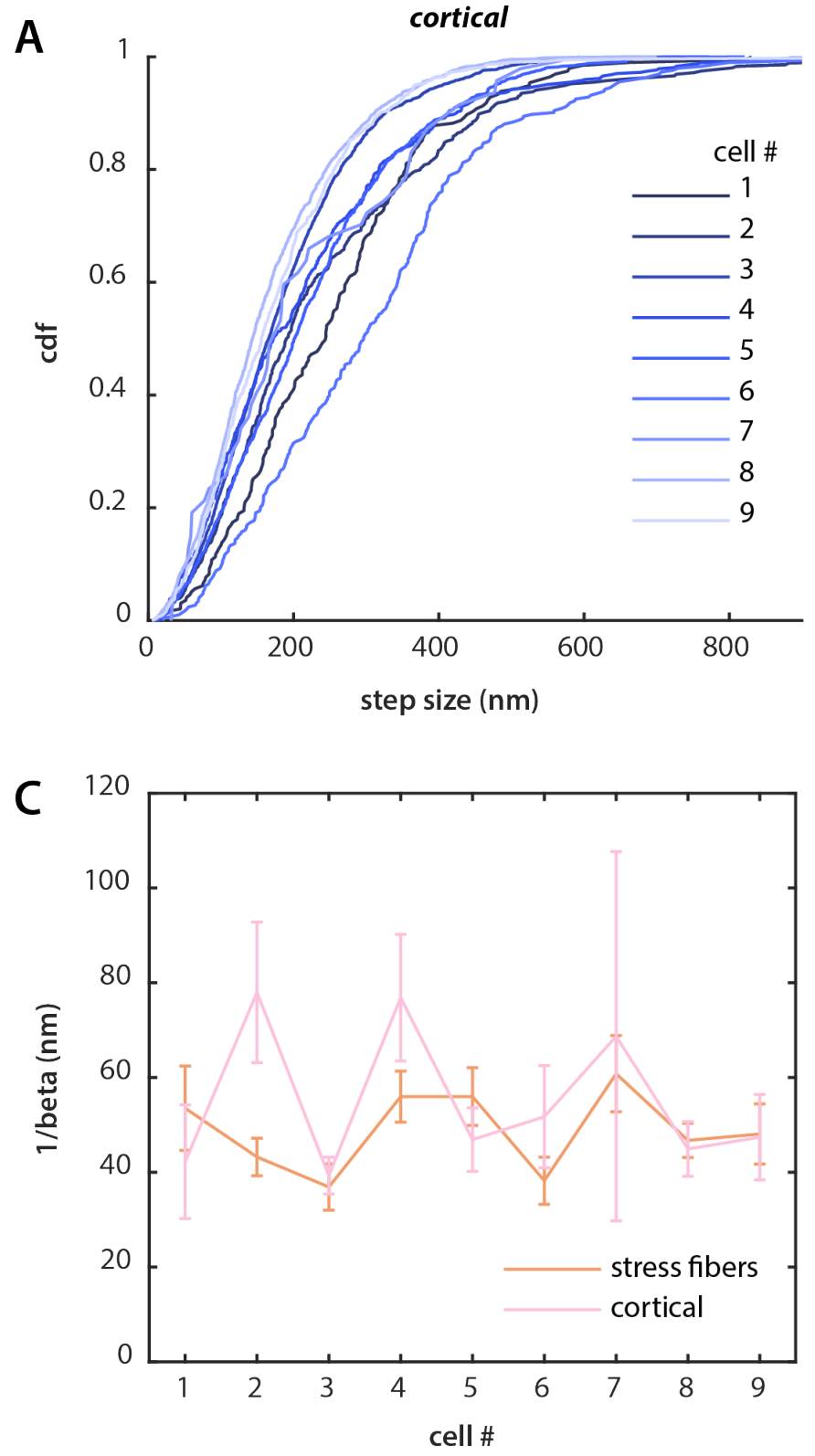
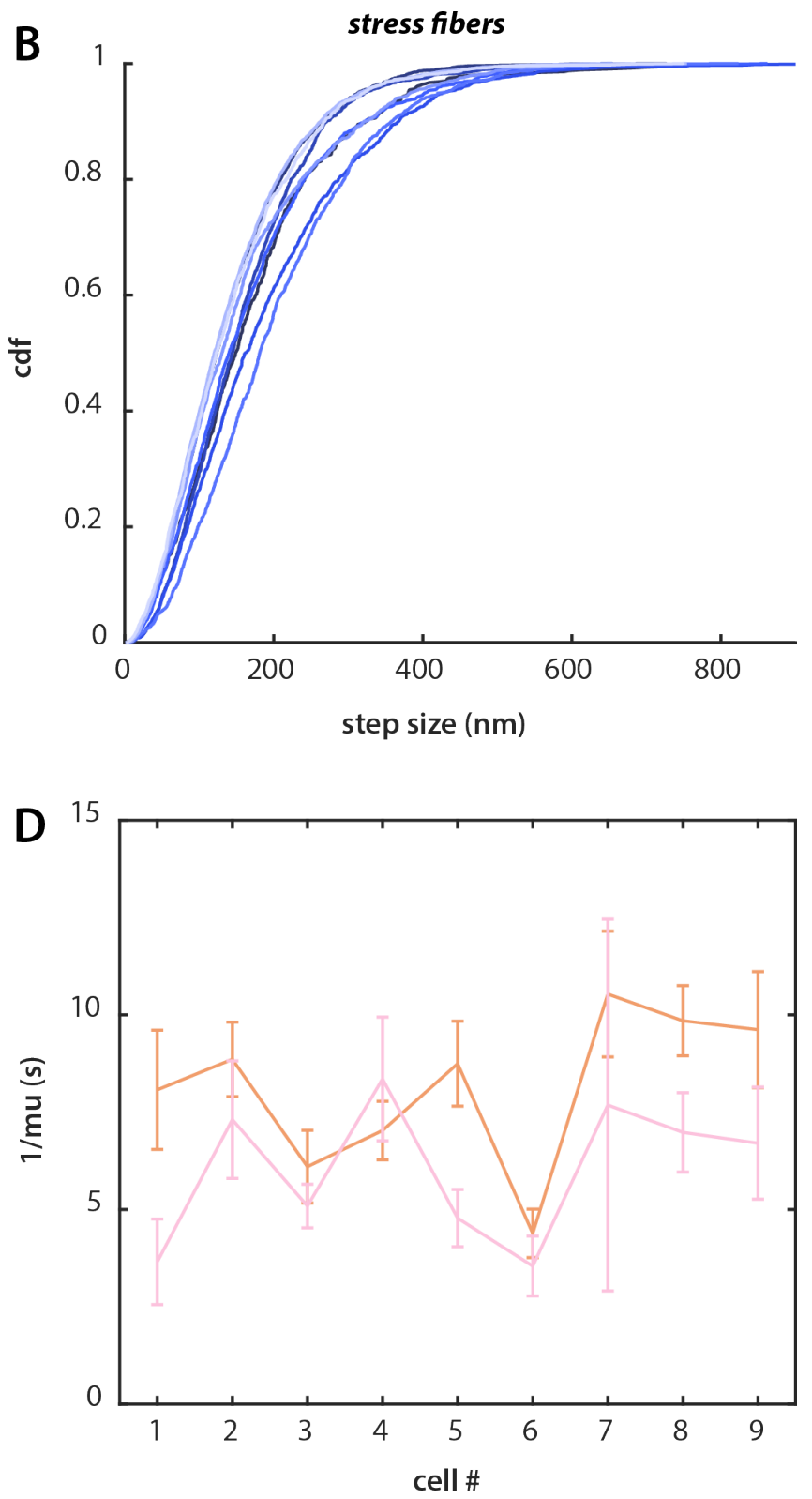

FIG. S5. A,B) Cumulative distribution functions of raw (uncorrected) step size distributions for cortical (A) and stress fiber (B) populations in HFFs, for nine separate cells. All cdfs are for a $20 \mathrm{~s}$ timescale. C,D) Best fit parameters for a jump process fit to the step size distributions in A and B. 

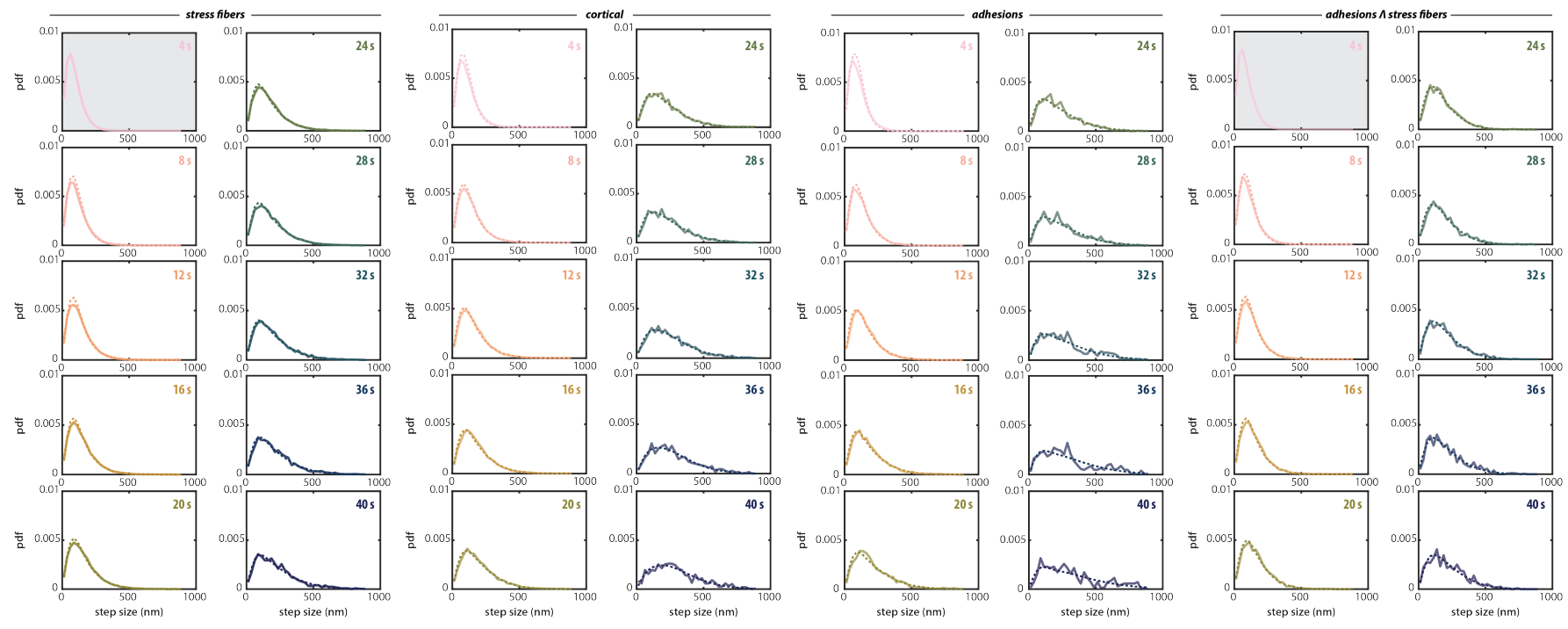

FIG. S6. Goodness of fit for jump process is good at most timescales for all four HFF F-actin populations. The measured distributions are shown in thick, semitransparent lines, and the best fits are shown in dashed lines. Fits did not converge for stress fiber populations (both with and without adhesions) at the $4 \mathrm{~s}$ timescale. 

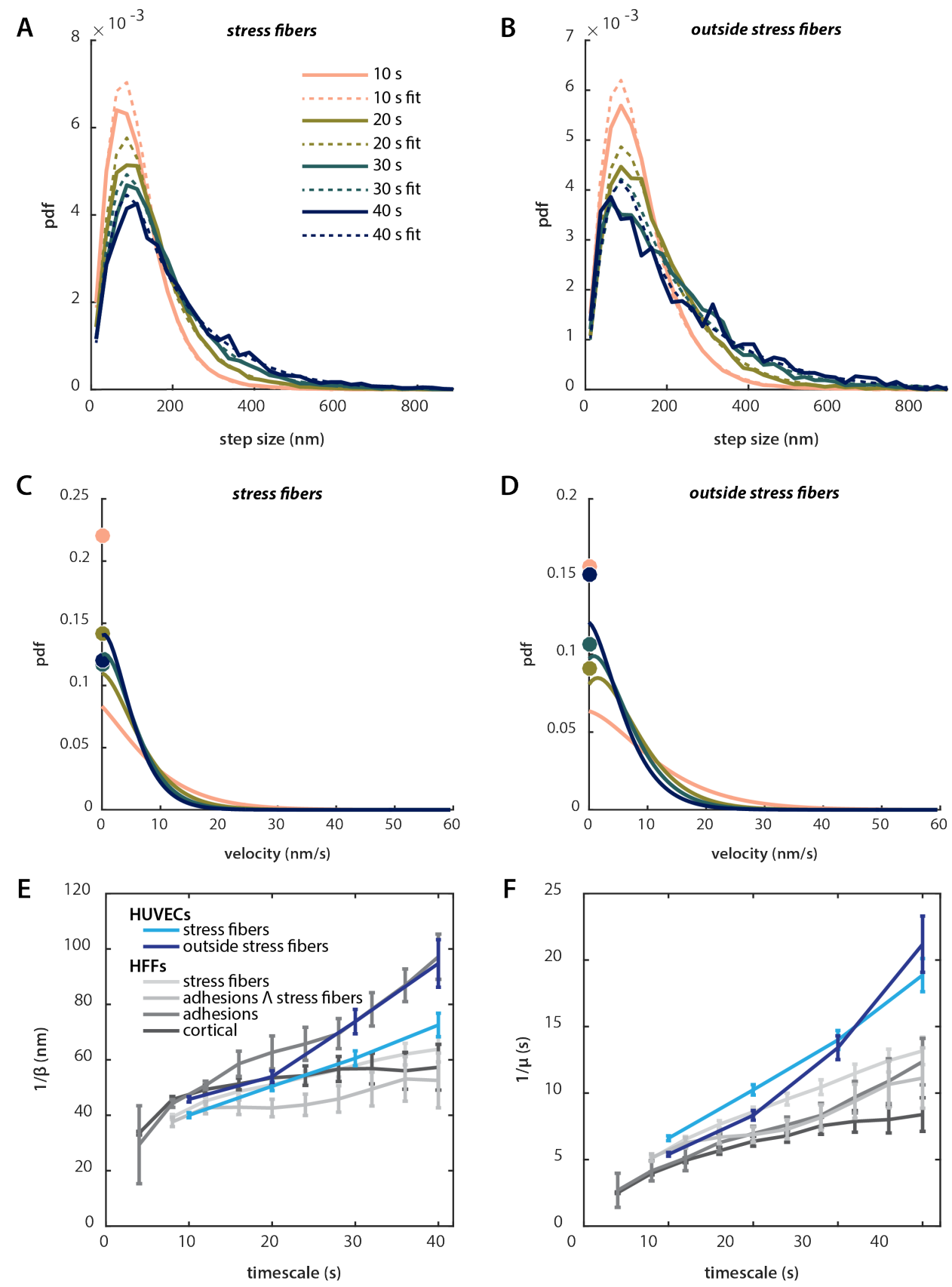

FIG. S7. A,B) Histograms for stress fibers (A) and outside stress fibers (B) in HUVECs, for varying timescales (10, 20, 30, $40 \mathrm{~s}$; solid lines), and the corresponding best fit for each (dashed lines). The outside stress fiber population may not exactly correspond to cortical actin in this case, as the high density of stress fibers in this cell type required lower labelling density. As such, many stress fibers are likely missed in the masking process. The outside stress fiber population is not well fit by the jump model, likely because it is a mixture of multiple populations. C,D) The inferred velocity probability distributions for the fits to the distributions in A and B. E,F) The best fit parameters for a jump process fit (shown in A-D) for HUVECs (blues), as compared to those for HFFs (grays) shows that HUVEC stress fibers share a similar jump distance $(1 / \beta)$ with HFF stress fibers, but have a longer wait time $(1 / \mu)$ between jumps. 
Video S1. An HFF labelled with a low dose of SiR-actin. Left: the whole cell is imaged every $2 \mathrm{~s}$ for 120 $\mathrm{s}$ total. The scale bar is $10 \mu \mathrm{m}$. Right: the two boxed regions of the cell on the left, magified $6 \mathrm{x}$. The scale bar is 1 $\mu \mathrm{m}$. 